\title{
Screening Isolates of Soybean mosaic virus for Infectivity in a Model Plant, Nicotiana benthamiana
}

L. Gao, R. Zhai, Y. K. Zhong, A. Karthikeyan, R. Ren, K. Zhang, K. Li, and H. J. Zhi, National Center for Soybean Improvement; Key Laboratory of Biology and Genetic Improvement of Soybean, Ministry of Agriculture, P.R. China; National Key Laboratory for Crop Genetics and Germplasm Enhancement; Nanjing Agricultural University, Weigang 1, Nanjing 210095, China

\begin{abstract}
Gao, L., Zhai, R., Zhong, Y. K., Karthikeyan, A., Ren, R., Zhang, K., Li, K., and Zhi, H. J. 2015. Screening isolates of Soybean mosaic virus for infectivity in a model plant, Nicotiana benthamiana. Plant Dis. 99:442-446.

Soybean mosaic virus (SMV), belonging to the genus Potyvirus of the family Potyviridae, has a relatively narrow host range almost exclusively confined to leguminous hosts. While disease management through genetic transformation can be an effective approach, soybean remains recalcitrant to routine genetic transformation. In this context, it is important to identify new hosts for SMV that can be used to develop effective transgenic resistance strategies. Transformation in Nicotiana benthamiana is simple and highly efficient; hence, here we demonstrate the infectivity of SMV strain SC7 in N. benthamiana plants. To identify an SMV strain infectious in $N$. benthamiana, we mechanically inoculated $N$. benthamiana plants with 37 isolates from 21 (SC1 to SC21) SMV strains. Plants

inoculated with isolates of strain SC7 produced mosaic symptoms on leaves. However, $N$. benthamiana plants inoculated with the 20 other SMV strains showed no visible symptoms. Furthermore, soybean cv. Nannong 1138-2 inoculated with sap prepared from symptomatic $N$. benthamiana leaves showed typical SMV mosaic symptoms 2 weeks after inoculation. In addition, SMV was detected in symptomatic $N$. benthamiana and soybean leaves by RT-PCR, DAS-ELISA, and further identified by sequencing. Together, the results indicate that $N$. benthamiana plants could support multiplication of SMV strain SC7. The findings of this study would be useful for the investigation of SMV resistance using the model plant $N$. benthamiana.
\end{abstract}

Soybean mosaic virus (SMV) is a threat to soybean production in most soybean [Glycine max (L.) Merr.] growing provinces of China. Infection by SMV usually results in severe yield losses and seed quality reductions. It has been reported that yield losses usually range from 8 to $50 \%$ under natural field conditions (14). SMV is a member of the genus Potyvirus in the family Potyviridae and it has been classified into 21 strains (SC1 to SC21) on the basis of responses and pathogenicity of SMV isolates on the uniform differentials system in China $(13,19,29,31,33)$. Because of the occurrence of SMV, improvement in production and productivity of soybean is becoming difficult and the disease is a major problem in China.

The increasing impact of SMV on soybean emphasizes the need to introduce SMV resistance into soybean in China. Genetically engineering soybean plants for resistance to viral pathogens has shown significant progress, which has paved the way for improving resistance to SMV in soybean. The most frequently employed methods for plant transformation are cotyledonary node-Agrobacterium-mediated and somatic embryo-particle-bombardment-mediated transformation (32). There have been some efforts to improve virus resistance in soybean by transgenic approaches. Overexpression of the SMV coat protein (CP) gene and the 3 '-untranslated region (UTR) resulted in high levels of resistance to SMV in transgenic soybean plants $(10,11,30)$. In addition, Di et al. (8) reported that transgenic soybean plants expressing the coat protein precursor (CP-P) gene of Bean pod mottle virus (BPMV) were resistant to BPMV, while two of the five $\mathrm{R}_{0}$ lines lost the CP-P gene in $R_{1}$ progenies, which could be due to the chimeric primary transformant or the unstable integration of the CP-P gene in the $\mathrm{R}_{0}$ plants.

Corresponding authors: H. J. Zhi and K. Li, E-mails: zhj@njau.edu.cn and kail@njau.edu.cn

Accepted for publication 13 October 2014.

http://dx.doi.org/10.1094/PDIS-04-14-0405-RE

(C) 2015 The American Phytopathological Society
Various methods have been used to improve soybean transformation. The preferred and highly reproducible transformation method uses cotyledonary nodes as the explant material for Agrobacteriummediated gene transfer with hygromycin B selection (24). The addition of thiol compounds to the cocultivation medium and the use of immature cotyledons also has improved soybean transformation efficiency (22-25). Thus, new methods have been developed for more efficient soybean transformation. There still remain, however, many challenges for genotype- and tissue-specific independent transformation of soybean (32).

In general, soybean genes were often evaluated for their function in heterogeneous plants such as Arabidopsis thaliana or tobacco (Nicotiana spp.) because soybean has remained recalcitrant to routine transformation. Furthermore, $N$. benthamiana is an ideal model plant for the investigation of the molecular basis of virus resistance genes and it has been successfully utilized in studying resistance genes to different plant viruses over the past 25 years $(5,12)$. Abel et al. (1) first developed transgenic $N$. tabacum resistance to Tobacco mosaic virus (TMV) through transferring TMV CP gene into tobacco. Prins et al. (26) obtained transgenic plants exhibiting high levels of resistance to Groundnut ring spot virus, Tomato chlorotic spot virus, and Tomato spotted wilt virus (TSWV) by transferring a vector comprising three viral nucleoprotein $(\mathrm{N})$ gene sequences into $N$. tabacum. Fuchs et al. (9) first reported that the transgenic $N$. benthamiana plants containing $\mathrm{CP}$ gene constructs of Watermelon mosaic virus2, Cucumber mosaic virus, and Zucchini yellow mosaic virus were resistant to these three viruses based on extensive field trials. Jan et al. (17) transformed the full-length CP gene of Turnip mosaic virus, which was linked to $\mathrm{N}$ gene segments of TSWV, into N. benthamiana, resulting in resistance to both of the viruses.

However, the host range of SMV is so narrow that the virus is almost completely confined to leguminous plants, such as soybean (G. max), common bean (Phaseolus vulgaris), cowpea (Vigna uniguiculata), and pea (Pisum sativum) $(3,4,16)$. In addition, some Chenopodium species, Senna occidentalis, and Pinellia ternata 
(Araceae) could be infected by SMV through mechanical or natural inoculation $(2,4,6,27,28)$. Nevertheless, there were no detailed reports of SMV infecting Nicotiana species naturally or artificially. Thus, the present study is aiming to infect $N$. benthamiana plants by SMV and results from these studies will be useful in SMV transgenic research.

\section{Materials and Methods}

Plant materials and SMV isolates. Soybean cultivar Nannong 1138-2 (highly susceptible to SMV), $N$. benthamiana, and 37 isolates of 21 SMV strains (SC1 to SC21; Table 1) were provided by the National Center for Soybean Improvement, Nanjing Agricultural University. Isolates were maintained in the susceptible Nannong 1138-2 and used to inoculate the $N$. benthamiana plants.

Virus inoculation. $N$. benthamiana seeds were germinated in vitro on $1 / 2 \mathrm{MS}$ medium $(21)$ at $25^{\circ} \mathrm{C}$ under 16 -h photoperiod in a growth chamber. After germination, seedlings were individually transplanted to plastic cups containing moistened nutrition soil mixed with vermiculite, and inoculated with SMV at the 5 to 6 leaf stage. The inocula were prepared from soybean plants infected with the 37 SMV isolates, and $N$. benthamiana seedlings were inoculated as described by Li et al. (19). Plants inoculated with $0.01 \mathrm{M}$ phosphate buffer were used as negative controls. All inoculated $N$. benthamiana plants were maintained in growth chambers with a 16-h photoperiod at $25^{\circ} \mathrm{C}$. Afterward, inoculated plants were observed at 5-day intervals for 4 weeks for virus symptoms. Four weeks after inoculations when the mosaic symptoms were clearly visible on leaves of $N$. benthamiana plants, symptomatic and asymptomatic leaves were collected and used for reverse transcription (RT)-PCR, double antibody sandwich (DAS)ELISA, and back-inoculation.

RT-PCR analysis. Total RNAs were extracted from all the samples (SMV-inoculated $N$. benthamiana plants), negative controls (mock-inoculated $N$. benthamiana plants), and positive controls (leaves of Nannong 1138-2 infected with SMV strain SC7) using a total RNA isolation kit (Tiangen, Beijing, China). The quality of the RNA was checked on $1.5 \%$ agarose gel. The extracted RNA was converted into cDNA using PrimeScript RT Master MixKit (Takara) according to the manufacturer's instructions. The first strand cDNA was used as a template for RT-PCR with the help of SMV CP gene-specific primers (expected amplicon size 1,058 bp). The forward (5'-ATGCTCAGACAAGTGAGCT-3') and reverse primers $\left(5^{\prime}\right.$-CTCCCTGCCATTCATAAAC- $\left.3^{\prime}\right)$ were designed based on the conserved region of SMV CP gene identified by aligning SMV sequences retrieved from the National Center for Biotechnology Information (NCBI) database. PCR amplification was conducted based on the instructions of $2 \times$ Taq PCR MasterMix (Tiangen, China). A volume of $25 \mu \mathrm{l}$ of PCR mixture contained $1 \mu \mathrm{l}$ of template cDNA $(<1 \mu \mathrm{g}), 1 \mu \mathrm{l}$ of each primer $(10 \mu \mathrm{M}), 12.5 \mu \mathrm{l}$ of $2 \times$ MasterMix, and $9.5 \mu \mathrm{l}$ of sterilized $\mathrm{dd}_{2} \mathrm{O}$. A thermal cycler (Perkin Elmer, Waltham, MA) was used and the conditions set at one cycle of $94^{\circ} \mathrm{C}$ for $5 \mathrm{~min}$; 35 cycles of $94^{\circ} \mathrm{C}$ for $30 \mathrm{~s}, 55^{\circ} \mathrm{C}$ for
$30 \mathrm{~s}$, and $72^{\circ} \mathrm{C}$ for $1 \mathrm{~min}$; one cycle of a final extension at $72^{\circ} \mathrm{C}$ for $5 \mathrm{~min}$; and a $4^{\circ} \mathrm{C}$ hold. The amplified products were separated by electrophoresis on a $1 \%$ agarose gel and photographed with a gel imaging system.

DAS-ELISA. Mock-inoculated and SMV-inoculated N. benthamiana plants were assayed for SMV infection by DAS-ELISA using kits purchased from ACD Inc. (cat \#V094-R1, Beijing, China) and following the manufacturer's instructions. To ensure reproducibility of DAS-ELISA, sample was collected from all the plants in every group for testing. The raw DAS-ELISA readings of each sample were averaged and converted to multiples of the negative controls (mock-inoculated plants). Samples with a relative ratio greater than 2.0 were considered positive for SMV.

Back-inoculation and sequencing. Inocula were prepared from symptomatic leaves of $N$. benthamiana plants (both RT-PCR and DAS-ELISA positive) and inoculated back to Nannong 1138-2 seedlings. Mock-inoculated plants were used as negative controls. After 2 weeks, when symptoms were clearly seen on the leaves, RT-PCR and DAS-ELISA (described earlier) were performed to confirm the presence of SMV. For further confirmation, CP gene fragments were purified by AxyPrep DNA Gel Extraction Kit (Axygen) and cloned into pMD 19-T vectors (Takara) according to the manufacturers' instructions and sequenced in Invitrogen Ltd., Shanghai, China.

\section{Results}

Symptoms on inoculated $N$. benthamiana plants. Only $N$. benthamiana plants inoculated with one strain, namely SC7 (isolates S9 and S10), showed distinct mosaic symptoms in systemic leaves. Yellow mosaic and light green mottled areas appeared on the leaves of $N$. benthamiana plants infected with this strain (Fig. 1). In contrast, $N$. benthamiana plants inoculated with the other 20 SMV strains were symptomless, and appeared healthy and similar to mockinoculated controls (Table 1). To corroborate the results, the experiments were repeated and similar results were obtained.

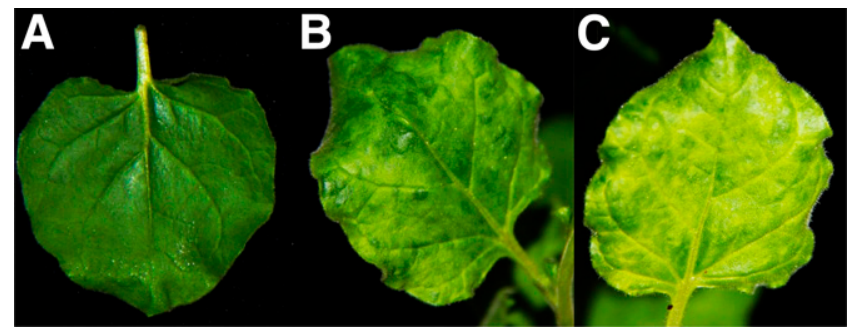

Fig. 1. SMV-like mosaic symptoms occurred on the leaves of Nicotiana benthamiana plants 4 weeks after inoculation. A, N. benthamiana inoculated with phosphate buffer as the negative control. B, Uninoculated upper leaves were symptomatic 4 weeks after the inoculation of isolate S9 of SMV strain SC7. C, Uninoculated upper leaves were symptomatic 4 weeks after the inoculation of isolate S10 of SMV strain SC7.

Table 1. SMV isolates used in study and the symptom appeared on Nicotiana benthamiana

\begin{tabular}{|c|c|c|c|c|c|c|c|c|}
\hline Strains & Isolates & $\begin{array}{c}\text { Symptom on } \\
\text { N. benthamiana }{ }^{a}\end{array}$ & Strains & Isolates & $\begin{array}{c}\text { Symptom on } \\
\text { N. benthamiana }{ }^{a}\end{array}$ & Strains & Isolates & $\begin{array}{c}\text { Symptom on } \\
N . \text { benthamiana }^{\text {a }}\end{array}$ \\
\hline SC1 & $\mathrm{S} 1$ & $-1-$ & SC10 & S15 & $-1-$ & SC17 & S29 & $-1-$ \\
\hline $\mathrm{SC} 2$ & $\mathrm{~S} 2$ & $-1-$ & & S16 & $-1-$ & SC18 & $\mathrm{S} 30$ & $-1-$ \\
\hline SC3 & $\mathrm{S} 3$ & $-1-$ & SC11 & S17 & $-1-$ & & S31 & $-1-$ \\
\hline \multirow[t]{2}{*}{$\mathrm{SC} 4$} & $\mathrm{~S} 4$ & $-1-$ & & S18 & $-1-$ & SC19 & S32 & $-1-$ \\
\hline & S5 & $-1-$ & SC12 & S19 & $-1-$ & & S33 & $-1-$ \\
\hline SC5 & S6 & $-1-$ & & $\mathrm{S} 20$ & $-1-$ & SC20 & S34 & $-1-$ \\
\hline \multirow[t]{2}{*}{ SC6 } & S7 & $-1-$ & SC13 & $\mathrm{S} 21$ & $-1-$ & & S35 & $-1-$ \\
\hline & S8 & $-1-$ & & $\mathrm{S} 22$ & $-1-$ & SC21 & S36 & $-1-$ \\
\hline \multirow[t]{2}{*}{ SC7 } & S9 & $-/ \mathrm{M}$ & SC14 & $\mathrm{S} 23$ & $-1-$ & & S37 & $-1-$ \\
\hline & S10 & $-/ \mathrm{M}$ & & $\mathrm{S} 24$ & $-1-$ & & & \\
\hline \multirow[t]{2}{*}{ SC8 } & S11 & $-1-$ & SC15 & $\mathrm{S} 25$ & $-1-$ & & & \\
\hline & $\mathrm{S} 12$ & $-1-$ & & S26 & $-1-$ & & & \\
\hline \multirow[t]{2}{*}{ SC9 } & S13 & $-1-$ & SC16 & $\mathrm{S} 27$ & $-1-$ & & & \\
\hline & S14 & $-1-$ & & S28 & $-1-$ & & & \\
\hline
\end{tabular}

\footnotetext{
a Symptoms on inoculated primary leaves/Symptom on uninoculated upper leaves; -: symptomless; M: mosaic.
} 
Detection of SMV in inoculated plants. The presence of viral RNA in inoculated $N$. benthamiana plants was studied by RTPCR using CP gene primers. Agarose gel electrophoresis of PCR products revealed the presence of the 1,058-bp CP amplicon only in $N$. benthamiana plants inoculated with two strain SC7 isolates (Fig. 2). No viral RNA was detected from the symptomless plants that were inoculated with the other 20 SMV strains. To confirm presence of SMV, the leaf samples were analyzed by DAS-ELISA, which showed that SMV was detected in $N$. benthamiana plants inoculated with SC7-S9 and SC7-S10 (Table 2). However, the SMV titers were below the detection limits for all the other SMV isolates.

Back-inoculation of soybean cv. Nannong 1138-2 and sequence analyses. Soybean cv. Nannong 1138-2 inoculated with sap prepared from symptomatic leaves of $N$. benthamiana plants developed typical mosaic symptoms on the trifoliate leaves two weeks after inoculation (Fig. 3). The accumulation of the viral RNA was confirmed by RT-PCR followed by DAS-ELISA (Fig. 4 and Table 3). Meanwhile, BLAST analyses showed that $\mathrm{CP}$ gene sequences (795 nucleotides in length) of SC7-S9 and SC7-S10 were the same and both of them had $97 \%$ nucleotide identities to SMV strain G3 (FJ640978) in the CP gene region. The detection

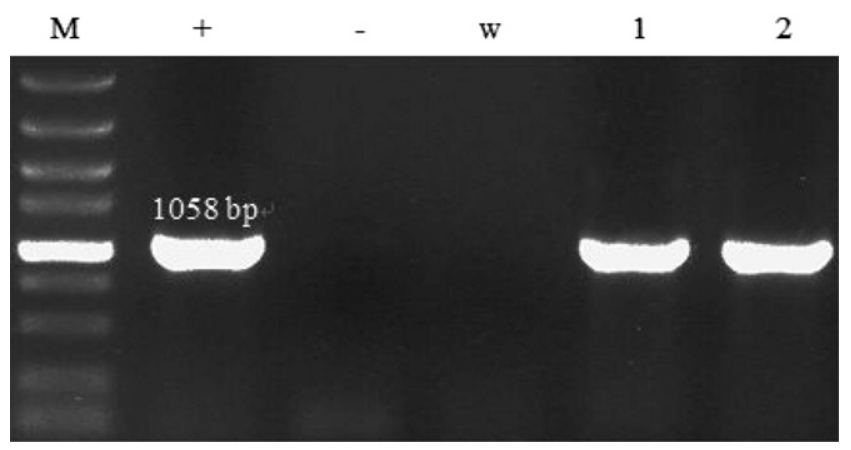

Fig. 2. Detection of SMV CP gene in SMV-inoculated Nicotiana benthamiana plants by RT-PCR. M, DNA marker DL5000 (Takara). +, positive control for SMV (Nannong 1138-2 infected with SMV strain SC7). -, negative control of mock-inoculated $N$. benthamiana plants. w, $N$. benthamiana plants without inoculation was the blank control. 1 and 2, N. benthamiana plants infected with isolate SC7-S9 and SC7-S10, respectively. of SMV in soybean plants after inoculation from symptomatic leaves of $N$. benthamiana and BLAST analyses both indicated that $N$. benthamiana plants had been infected by SMV through artificial inoculation.

\section{Discussion}

Since the production of the first transgenic soybean plants $(15,20)$, a variety of methods for generating transgenic virus resistance have been developed for soybean. However, almost all of the SMVresistant transgenic soybean lines have been developed using the SMV CP gene. The observed resistance was attributed to overexpression of the SMV CP or RNA interference $(10,11,18,30)$. Other SMV genes or sequences have not been transformed. At present, transformation efficiency of soybean is still low. The transgenic resistance for plant viruses has been successfully developed using Nicotiana species with high rates of success. Especially, $N$. benthamiana has become the most widely used experimental host in plant viruses due mainly to the susceptibility to a wide variety of plant viruses and can be genetically transformed with high regeneration frequency $(5,12)$. However, the host range of SMV is limited and there were no detailed reports of SMV infecting Nicotiana species naturally or artificially. Thus, it is almost impossible to evaluate resistance candidate genes to SMV through transgenic $N$. benthamiana plants. It is worth mentioning that Christie and Crawford (7) as well as Almeida et al. (2) reported infectivity of SMV isolates on the $N$. benthaminana. However, the reports were not detailed and there were no further analyses of the SMV-inoculated $N$. benthaminana plants, and the strains/isolates used in study were also limited. In our study, we used

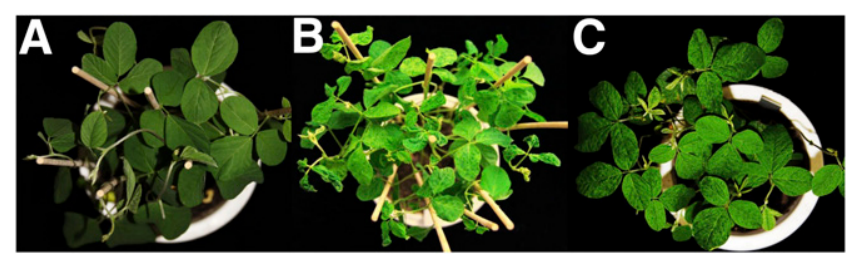

Fig. 3. Soybean cv. Nannong 1138-2 showed typical mosaic symptom 2 weeks after back-inoculation. A, Mock-inoculated Nannong 1138-2 as the negative control. B, Back-inoculation with symptomatic leaves of $N$. benthamiana plants infected with SC7-S9. C, Back-inoculation with the symptomatic leaves of $N$. benthamiana plants infected with SC7-S10.

Table 2. Serological determinations of DAS-ELISA of SMV-inoculated Nicotiana benthamiana plants based on the optical density $\left(\mathrm{OD}_{405 \mathrm{~nm}}\right)^{\mathrm{a}}$

\begin{tabular}{|c|c|c|c|c|c|c|c|}
\hline Strains & Isolates & $P^{b}\left(O D_{405 n m}\right)$ & $\mathbf{P} / \mathbf{N}^{\mathbf{c}}$ & Strains & Isolates & $P^{b}\left(O D_{405 n m}\right)$ & $\mathbf{P} / \mathbf{N}^{\mathbf{c}}$ \\
\hline $\mathrm{SC} 1$ & S1 & 0.118 & $1.00(-)$ & & S20 & 0.121 & $1.03(-)$ \\
\hline $\mathrm{SC} 2$ & S2 & 0.115 & $0.98(-)$ & $\mathrm{SC} 13$ & S21 & 0.115 & $0.97(-)$ \\
\hline $\mathrm{SC} 3$ & S3 & 0.116 & $0.98(-)$ & & S22 & 0.114 & $0.97(-)$ \\
\hline \multirow[t]{2}{*}{$\mathrm{SC} 4$} & S4 & 0.113 & $0.96(-)$ & SC14 & S23 & 0.128 & $1.08(-)$ \\
\hline & S5 & 0.118 & $1.00(-)$ & & S24 & 0.113 & $0.96(-)$ \\
\hline SC5 & S6 & 0.115 & $0.97(-)$ & SC15 & S25 & 0.113 & $0.96(-)$ \\
\hline \multirow[t]{2}{*}{ SC6 } & S7 & 0.139 & $1.18(-)$ & & S26 & 0.081 & $0.69(-)$ \\
\hline & S8 & 0.116 & $0.98(-)$ & SC16 & S27 & 0.132 & $1.12(-)$ \\
\hline \multirow[t]{2}{*}{$\mathrm{SC} 7$} & S9 & 3.353 & $28.42(+)$ & & $\mathrm{S} 28$ & 0.112 & $0.95(-)$ \\
\hline & S10 & 3.380 & $28.64(+)$ & SC17 & S29 & 0.117 & $0.99(-)$ \\
\hline \multirow[t]{2}{*}{ SC8 } & S11 & 0.116 & $0.98(-)$ & SC18 & S30 & 0.113 & $0.96(-)$ \\
\hline & S12 & 0.115 & $0.98(-)$ & & S31 & 0.113 & $0.96(-)$ \\
\hline \multirow[t]{2}{*}{ SC9 } & S13 & 0.114 & $0.97(-)$ & SC19 & S32 & 0.117 & $0.99(-)$ \\
\hline & S14 & 0.116 & $0.98(-)$ & & S33 & 0.115 & $0.97(-)$ \\
\hline \multirow[t]{2}{*}{ SC10 } & S15 & 0.125 & $1.06(-)$ & SC20 & S34 & 0.115 & $0.97(-)$ \\
\hline & S16 & 0.110 & $0.93(-)$ & & S35 & 0.115 & $0.97(-)$ \\
\hline \multirow[t]{2}{*}{$\mathrm{SC} 11$} & S17 & 0.113 & $0.96(-)$ & $\mathrm{SC} 21$ & S36 & 0.109 & $0.92(-)$ \\
\hline & S18 & 0.113 & $0.96(-)$ & & S37 & 0.116 & $0.98(-)$ \\
\hline $\mathrm{SC} 12$ & S19 & 0.122 & $1.03(-)$ & & & & \\
\hline
\end{tabular}

a $(+)$ : positive for SMV; (-): negative for SMV.

b $\mathrm{OD}_{405 \mathrm{~nm}}$ value of each sample was calculated by averaging the three readings of the plate.

${ }^{c} \mathrm{OD}_{405 \mathrm{~nm}}$ value of negative control was calculated by averaging the three readings of the plate and it is 0.118 . 


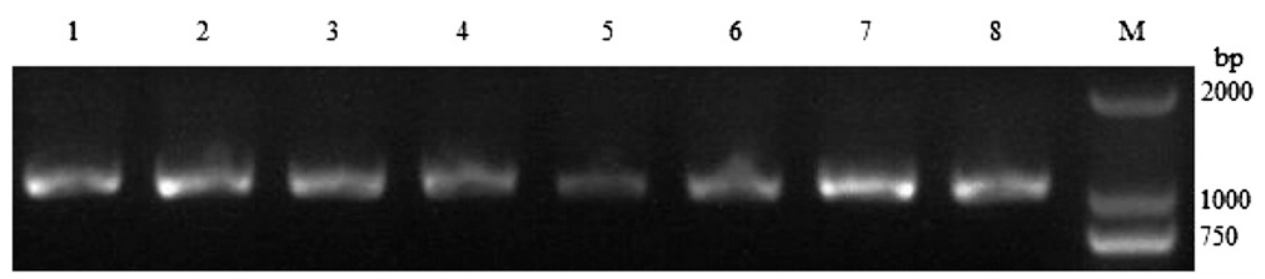

Fig. 4. Detection of SMV CP gene in back-inoculated soybean cv. Nannong 1138-2 by RT-PCR. M, DNA marker 2000 (Tiangen, China). 1 to 4, Nannong 1138-2 inoculated back with symptomatic leaves of N. benthamiana plants infected with the isolate of SC7-S9. 5 to 8, Nannong 1138-2 inoculated back with symptomatic leaves of N. benthamiana plants infected with the isolate of SC7-S10.

Table 3. DAS-ELISA analyses of the back-inoculated soybean based on the optical density value $\left(\mathrm{OD}_{405 \mathrm{~nm}}\right)^{\mathrm{a}}$

\begin{tabular}{lccccc}
\hline & \multicolumn{2}{c}{ SC7-S9 } & & \multicolumn{2}{c}{ SC7-S10 } \\
\cline { 2 - 3 } Back inoculation & $\mathbf{P}^{\mathbf{b}}\left(\mathbf{D}_{\mathbf{4 0 5 n m}}\right)$ & $\mathbf{P} / \mathbf{N}^{\mathbf{c}}$ & & $\mathbf{P}^{\mathbf{b}}\left(\mathbf{D}_{\mathbf{4 0 5 n m}}\right)$ & $\mathbf{P} / \mathbf{N}^{\mathbf{c}}$ \\
\hline 1 & 3.719 & $22.54(+)$ & & 3.056 & $18.52(+)$ \\
2 & 3.664 & $22.21(+)$ & & 3.677 & $22.28(+)$ \\
3 & 3.220 & $19.52(+)$ & & 3.714 & $22.51(+)$ \\
4 & 3.388 & $20.53(+)$ & & 3.653 & $22.14(+)$ \\
\hline
\end{tabular}

a $(+)$ : positive for SMV.

b $\mathrm{OD}_{405 \mathrm{~nm}}$ value of each back-inoculation was calculated by averaging the three readings of the plate.

c $\mathrm{OD}_{405 \mathrm{~nm}}$ value of negative control was calculated by averaging the three readings of the plate and it is 0.165 .

37 isolates of 21 SMV strains (SC1 to SC21) and proved N. benthaminana to be infected by visual symptoms, RT-PCR, DAS-ELISA, and back-inoculation, as well as by sequencing of $\mathrm{CP}$ gene of SMV strain SC7.

In this study, 37 isolates of 21 SMV strains were used to inoculate the $N$. benthamiana plants. Interestingly, among them only two isolates S9 and S10 of SMV strain SC7 induced mosaic symptoms on $N$. benthamiana leaves. The remaining isolates did not infect $N$. benthamiana. As is known, leguminous plants are the main host of SMV, but the virus also infects some other plants such as Pinellia ternata, Senna occidentalis, and some Chenopodium species naturally or artificially $(2,4,6)$. Previously, Chen et al. (6) reported that $P$. ternata plants could be infected by SMV isolate P-1 naturally. However, P. ternata remained symptomless and no virus was detectable when inoculated with SMV isolate HH5. On the other hand, although isolate P-1 could infect soybean and P. ternata, it failed to infect $N$. benthamiana, $N$. tabacum, Chenopodium amaranticola, and other plants. As well, some Chenopodium species could be infected by SMV artificially $(4,6)$.

Results from the study clearly show that $N$. benthamiana is a host for SMV strain SC7, and that the SC7 strain is different from the other strains tested here. Therefore, investigating the factors affecting host range of SC7 and studying the similarity of nucleotide sequences of other SC7 isolates will be useful to understand the evolutionary and taxonomic implications between SC7 and other SMV strains. It is worth mentioning that as more and more SMV SC7 isolates are sequenced, the complete genome sequences and partial gene sequences can be used to study the phylogenetic relationship and molecular variability of the isolates. The outcomes of these studies will certainly provide new insights into the evolutionary process of SMV in relation to its natural host range.

In conclusion, to the best of our knowledge, this is the first detailed report of SMV infecting $N$. benthamiana. Results from this research will enable significant progress in the investigation of the molecular basis of SMV transgenic resistance using the model plant $N$. benthamiana.

\section{Acknowledgments}

This work was supported by the National Natural Science Foundation of China (Grant No.31171574, 31371646, 31101164), the National Soybean Industrial Technology System of China (No. CARS-004), the Fund of Transgenic Breeding for Soybean Resistance to Soybean mosaic virus (No.2008ZX08004-004), the 111 project (B08025), and PAPD.

\section{Literature Cited}

1. Abel, P. P., Nelson, R. S., De, B., Hoffmann, N., Rogers, S. G., Fraley, R. T., and Beachy, R. N. 1986. Delay of disease development in transgenic plants that express the Tobacco mosaic virus coat protein gene. Science 232: 738-743.

2. Almeida, A. M. R., Sakai, J., Souto, E. R., Kitajima, E. W., Fukuji, T. S., and Hanada, K. 2002. Mosaic in Senna occidentalis in Southern Brazil induced by a new strain of Soybean mosaic virus. Fitopatol. Bras. 27:151-156.

3. Bos, L. 1972. Soybean mosaic virus. CMI/AAB Description of Plant Viruses 93:49-52.

4. Boswell, K. F., and Gibbs, A. J. 1983. Viruses of legumes. Descriptions and keys from VIDE. Canberra. Aust. Natl. Univ. Res. Sch. Biol. Sci. 139:77-107.

5. Chakrabarty, R., Banerjee, R., Chung, S. M., Farman, M., Citovsky, V., Hogenhout, S. A., Tzfira, T., and Goodin, M. M. 2007. pSITE vectors for stable integration or transient expression of autofluorescent protein fusions in plants: probing Nicotiana benthamiana-virus interactions. Mol. Plant Microbe Interact. 20:740-750.

6. Chen, J., Zheng, H. Y., Lin, L., Adams, M. J., Antoniw, J. F., Zhao, M. F., Shang, Y. F., and Chen, J. P. 2004. A virus related to Soybean mosaic virus from Pinellia ternata in China and its comparison with local soybean SMV isolates. Arch. Virol. 149:349-363.

7. Christie, S. R., and Crawford, W. E. 1978. Plant virus range of Nicotiana benthamiana. Plant Dis. Report. 62:20-22.

8. Di, R., Purcell, V., Collins, G. B., and Ghabrial, S. A. 1996. Production of transgenic soybean lines expressing the Bean pod mottle virus coat protein precursor gene. Plant Cell Rep. 15:746-750.

9. Fuchs, M., McFerson, J. R., Tricoli, D. M., McMaster, J. R., Deng, R. Z., Boeshore, M. L., Reynolds, J. F., Russell, P. F., Quemada, H. D., and Gonsalves, D. 1997. Cantaloupe line CZW-30 containing coat protein genes of Cucumber mosaic virus, Zucchini yellow mosaic virus, and Watermelon mosaic virus -2 is resistant to these three viruses in the field. Mol. Breed. 3: 279-290.

10. Furutani, N., Hidaka, S., Kosaka, Y., Shizukawa, Y., and Kanematsu, S. 2006. Coat protein gene-mediated resistance to Soybean mosaic virus in transgenic soybean. Breed. Sci. 56:119-124.

11. Furutani, N., Yamagishi, N., Hidaka, S., Shizukawa, Y., Kanemastu, S., and Kosaka, Y. 2007. Soybean mosaic virus resistance in transgenic soybean caused by post-transcriptional gene silencing. Breed. Sci. 57:123-128.

12. Goodin, M. M., Zaitlin, D., Naidu, R. A., and Lommel, S. A. 2008. Nicotiana benthamiana: its history and future as a model for plant-pathogen interactions. Mol. Plant Microbe Interact. 21:1015-1026.

13. Guo, D. Q., Zhi, H. J., Wang, Y. W., Gai, J. Y., Zhou, X. A., Yang, C. L., Li, K., and Li, H. C. 2005. Identification and distribution of Soybean mosaic virus strains in Middle and Northern Huang-Huai Region of China. Chin. J. Oil Crop Sci. 27:64-68.

14. Hill, J. H. 1999. Soybean mosaic virus. Pages 70-71. in: Compendium of Soybean Diseases, 4th Ed., G. L. Hartman, J. B. Sinclair, and J. C. Rupe, eds. The American Phytopathological Society, St. Paul, MN.

15. Hinchee, M. A., Connor-Ward, D. V., Newell, C. A., McDonnell, R. E., Sato, S. J., Gasser, C. S., Fischhoff, D. A., Re, D. B., Fraley, R. T., and Horsch, R. B. 1988. Production of transgenic soybean plants using Agrobacterium-mediated DNA transfer. Nat. Biotechnol. 6:915-922.

16. Hunst, P. L., and Tolin, S. A. 1982. Isolation and comparison of two strains of Soybean mosaic virus. Phytopathology 72:710-713.

17. Jan, F. J., Fagoaga, C., Pang, S. Z., and Gonsalves, D. 2000. A single chimeric transgene derived from two distinct viruses confers multi-virus resistance in transgenic plants through homology-dependent gene silencing. J. Gen. Virol. 81:2103-2109.

18. Kim, H. J., Kim, M. J., Pak, J. H., Jung, H. W., Choi, H. K., Lee, Y. H., Baek, I. Y., Ko, J. M., Jeong, S. C., Pack, I. S., Ryu, K. H., and Chung, Y. S. 2013. Characterization of SMV resistance of soybean produced by genetic transformation of SMV-CP gene in RNAi. Plant Biotechnol. Rep. 7:425-433. 
19. Li, K., Yang, Q. H., Zhi, H. J., and Gai, J. Y. 2010. Identification and distribution of Soybean mosaic virus strains in Southern China. Plant Dis. 94:351-357.

20. McCabe, D. E., Swain, W. F., Martinell, B. J., and Christou, P. 1988. Stable transformation of soybean (Glycine max) by particle acceleration. Nat. Biotechnol. 6:923-926.

21. Murashige, T., and Skoog, F. 1962. A revised medium for rapid growth and bioassays with tobacco tissue cultures. Physiol. Plant. 15:473-497.

22. Olhoft, P. M., Lin, K., Galbraith, J., Nielsen, N. C., and Somers, D. A. 2001. The role of thiol compounds in increasing Agrobacterium-mediated transformation of soybean cotyledonary-node cells. Plant Cell Rep. 20: 731-737.

23. Olhoft, P. M., and Somers, D. A. 2001. L-Cysteine increases Agrobacteriummediated T-DNA delivery into soybean cotyledonary-node cells. Plant Cell Rep. 20:706-711.

24. Olhoft, P. M., Flagel, L. E., Donovan, C. M., and Somers, D. A. 2003. Efficient soybean transformation using hygromycin B selection in the cotyledonary-node method. Planta 216:723-735.

25. Paz, M. M., Martinez, J. C., Kalvig, A. B., Fonger, T. M., and Wang, K. 2006. Improved cotyledonary node method using an alternative explant derived from mature seed for efficient Agrobacterium-mediated soybean transformation. Plant Cell Rep. 25:206-213.

26. Prins, M., De Haan, P., Luyten, R., Van Veller, M., Van Grinsven, M., and Goldbach, R. 1995. Broad resistance to tospoviruses in transgenic tobacco plants expressing three tospoviral nucleoprotein gene sequences. Mol. Plant Microbe Interact. 8:85-91.

27. Shi, Y. H., Hong, X. Y., Chen, J., Adams, M. J., Zheng, H. Y., Lin, L., Qin, B. X., and Chen, J. P. 2005. Further molecular characterisation of potyviruses infecting aroid plants for medicinal use in China. Arch. Virol. 150:125-135.

28. Sun, H. H., Shen Tu, S. S., Xue, F., Duns, G., and Chen, J. S. 2008. Molecular characterization and evolutionary analysis of Soybean mosaic virus infecting Pinellia ternata in China. Virus Genes 36:177-190.

29. Wang, X. Q., Gai, J. Y., and Pu, Z. Q. 2003. Classification and distribution of strain groups of Soybean mosaic virus in Middle and Lower Huang-Huai and Changjiang Valleys. Soybean Sci. 22:102-107.

30. Wang, X. Y., Eggenberger, A. L., Nutter, F. W., Jr., and Hill, J. H. 2001 Pathogen-derived transgenic resistance to Soybean mosaic virus in soybean. Mol. Breed. 8:119-127.

31. Wang, Y. W., Zhi, H. J., Guo, D. Q., Gai, J. Y., Chen, Q. S., Li, K., and Li, H. C. 2004. Classification and distribution of strain groups of Soybean mosaic virus in Northern China spring planting soybean region. Soybean Sci. 24:263-268.

32. Yamada, T., Takagi, K., and Ishimoto, M. 2012. Recent advances in soybean transformation and their application to molecular breeding and genomic analysis. Breed. Sci. 61:480-494.

33. Zhan, Y., Zhi, H. J., Yu, D. Y., and Gai, J. Y. 2005. Identification and distribution of SMV strains in Huang-Huai Valleys. Scientia Agric. Sin. 39: 2009-2015. 